\title{
Design and performance assessment of an underactuated hand for industrial applications
}

\author{
C. Meijneke, G. A. Kragten, and M. Wisse \\ Faculty of Mechanical, Maritime and Materials Engineering, Dept. of BioMechanical Engineering, \\ Delft University of Technology, Mekelweg 2, 2628 CD Delft, The Netherlands
}

Received: 27 February 2010 - Revised: 1 July 2010 - Accepted: 19 August 2010 - Published: 8 February 2011

\begin{abstract}
The Delft Hand 2 (DH-2) is an underactuated robot hand meant for industrial applications, having six degrees of freedom (DoF), one actuator (DoA) and no sensors. It was designed to provide a cheap and robust hand to grasp a large range of objects without damaging them. The goal of this paper is to assess the design and performance of the DH-2, demonstrating how the design was optimized for its intended application area and how the hand was simplified to make it commercially attractive. Performance tests show that the DH-2 has a payload of $2 \mathrm{~kg}$ for an object range of 60 to $120 \mathrm{~mm}$, it can close or open within $0.5 \mathrm{~s}$, and it only uses open-loop control by means of the input voltage of the motor. The results demonstrate that the industrial need of a simple, cheap and effective robotic hand can be achieved with the principle of underactuation and the use of conventional components.
\end{abstract}

This paper was presented at the IFToMM/ASME International Workshop on Underactuated Grasping (UG2010), 19 August 2010, Montréal, Canada.

\section{Introduction}

The principle of underactuation (Birglen et al., 2008) can make a robotic hand adaptive to the shape of objects, enabling it to grasp a diversity of objects. The demand of grasping various objects can be found in, for example, service robots for grasping in household environments, or sorting systems in industry. Although a lot of robot hands were made using this principle, only very few are commercially available (i.e., the Barrett hand (Townsend, 2000) and the SARAH (Laliberté and Gosselin, 2001)). This small number might be caused by unfamiliarity with underactuation, or because the solutions are still too complex or too expensive. To get the industry interested, there is a need of cheap, simple and robust solutions.

In 2008, an underactuated hand - called Delft Hand 1 (Meijneke and Wilbers, 2009) - was built at Delft University of Technology. The objectives were to learn about the performance of underactuated grasping, and to get industry interested. Although the majority of feedback from the industry was positive, issues like closing speed of the hand, the

Correspondence to: C. Meijneke (c.meijneke@tudelft.nl) robustness of the design and the grasp reliability needed to be improved.

To address these issues, a second prototype - called Delft Hand 2 (DH-2) - was developed. Figure 1 shows the design of this hand featuring three similar fingers consisting of two phalanges, which are all driven by one actuator without any sensory feedback.

The objectives of this paper are firstly to assess the critical design choices of the DH-2 leading to a simple, cheap and robust hand. Secondly, the performance of the DH-2 is assessed. The critical performance factors, according to industrial partners, are the (1) payload, (2) the closing and opening time of the hand, (3) and the range of object sizes which can be grasped by the hand. Simple and robust means that the number of actuators and sensors is minimized and external disturbances can be resisted.

The structure of this paper is as follows. In Sect. 2 the design requirements are defined, and the conceptual and dimensional design choices are assessed based on performance calculations. Section 3 describes the experiments to measure the payload for a range of object sizes, the relation between the motor voltage (input) and the contact forces on objects, and the closing or opening time of the hand. The results of these experiments are given in Sect. 4, followed by Discussion and Conclusion. 


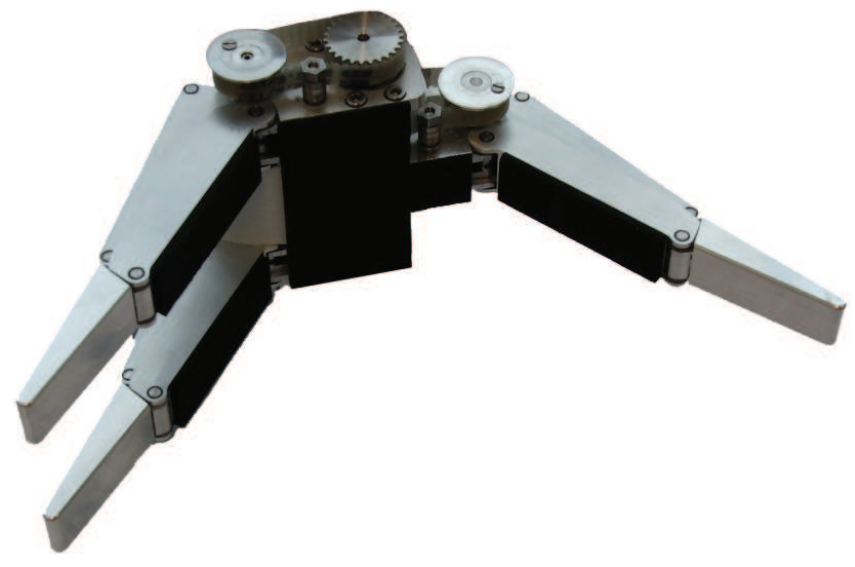

Figure 1. Picture of the DH-2 prototype.

\section{Design}

This section shows the main requirements for the design of the DH-2 prototype and the design choices made to satisfy these requirements. The model used to optimize the performance will be discussed and some predictions of the performance are made.

\subsection{Requirements}

The following functional requirements were defined based on interviews with industrial partners involved in areas like distribution centers and fruit and vegetable sorting: (1) a minimum payload of $2 \mathrm{~kg}$, (2) a maximal closing time of $0.5 \mathrm{~s}$, and (3) a product range of $60 \mathrm{~mm}$ to $120 \mathrm{~mm}$ diameter. The weight of most products were much smaller than $2 \mathrm{~kg}$, but accelerations in pick and place tasks increase the required payload. The cycle time to do profitable pick and place tasks determined the time to fully open and close the grasper, which had to be less then $0.5 \mathrm{~s}$. The graspable range refers to the diameter $\left(D_{\text {obj }}\right)$ of a (long) cylindrical object, because the majority of the target products approximates this shape. We consider an object to be graspable when the robot hand can successfully fix the object in a power grasp posture (Napier, 1956).

\subsection{Mechanical design}

In DH-2 the motor torque is distributed between the fingers and phalanges by means of differential mechanisms. To ensure robustness, a belt transmission and a gear differential were chosen in the DH-2 to distribute the motor torque to the three fingers. Each finger consists of two phalanges driven by a four-bar linkage mechanism as inspired by the MARS and SARAH (Laliberté and Gosselin, 2001). To prevent entrapment of objects between the linkages, the parts of the fingers where designed to form a closed casing (Fig. 2). When the

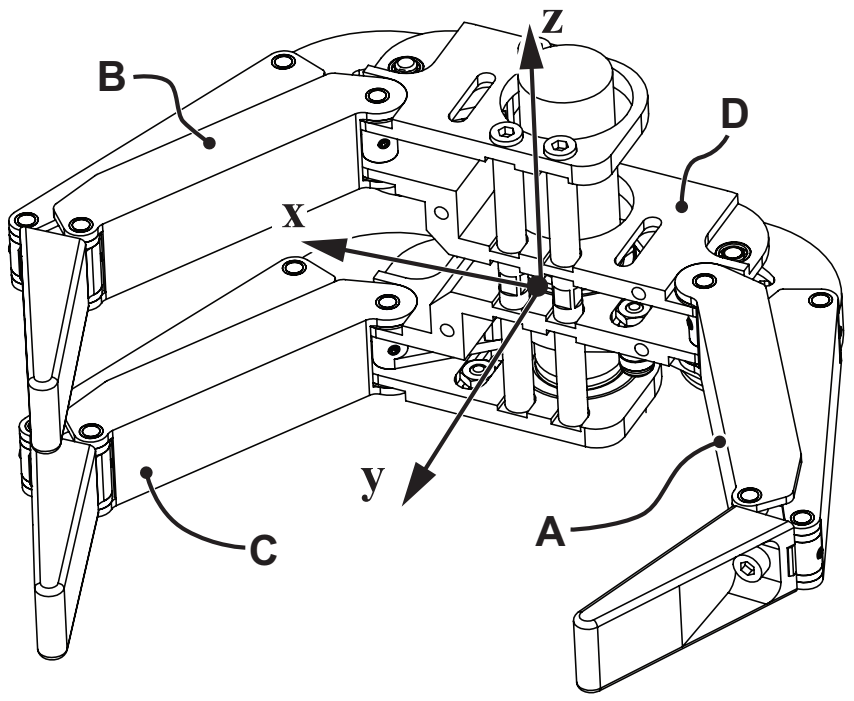

Figure 2. Design drawing of DH-2, with one finger A, two opposite fingers $\mathrm{B}$ and $\mathrm{C}$ and a palm $\mathrm{D}$.

closing and opening sequence of the phalanges are critical, linear springs can be mounted in the finger casings.

To obtain a simple and cheap design, we minimized the amount of mechanical and electronic parts. It was therefore decided not to use any force or position sensor. In addition. the fingers can be both opened and closed by only one Maxon RE13 3.5W motor (with a GP13 67:1 gearbox). When a voltage of $24 \mathrm{~V}$ is applied to the motor this results in a constant actuation torque of $1 \mathrm{Nm}$ applied at the base of finger $\mathrm{A}$ and $0.5 \mathrm{Nm}$ on each of the opposite fingers B and C (Fig. 2) as defined in (Birglen et al., 2008, p. 35). Hence, only two cables for the power supply to the motor were required. This approach also provided the possibility to fit the full mechanism in a palm of $30 \mathrm{~mm}$ thickness and $75 \mathrm{~mm}$ width.

The absence of sensory feedback implies open-loop control of the hand. It was chosen to control the contact forces of the hand simply by the input voltage to the motor. The relation between this input voltage and contact forces is experimentally assessed in the next chapter.

\subsection{Dimensional design}

The main dimensions of the linkage mechanism of the fingers and the width of the palm were determined by performance calculations according to the model proposed in Kragten and Herder (2010). In this model the equilibrium configuration and the contact forces of the fingers were calculated as a function of the objects size and object position. Friction in the mechanism of the fingers and between the fingers and the object was neglected in this model. A contact point between a phalanx and the object can be geometrically described by a loop closure vector equation, as shown in Fig. 3. For the 


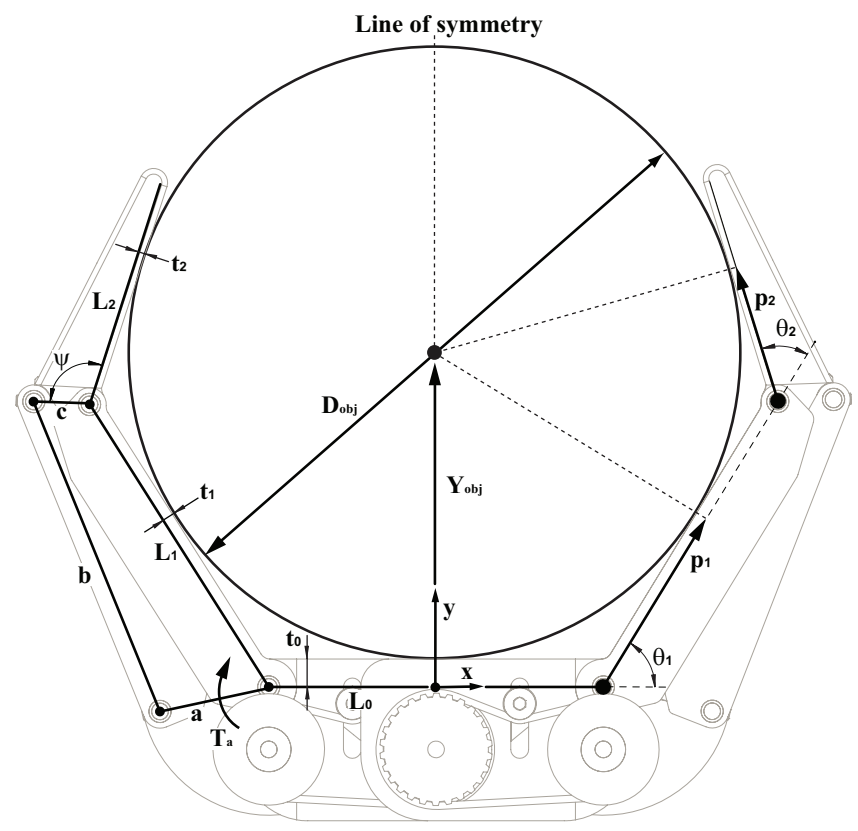

Figure 3. Schematic representation of a symmetric power grasp of the DH-2 on a cylindrical object of radius $r_{\mathrm{obj}}$ and position $\left(X_{\mathrm{obj}}, Y_{\mathrm{obj}}\right)$. On the left finger, the length of the phalanges $L_{i}$, the dimensions of the linkage mechanism $a, b, c$, the angle $\psi$ between the distal phalanx $L_{2}$ and link $c$, the thickness of the phalanges $t_{i}$, and the actuation torque $T_{a}$ are shown. On the right finger the contact point position on the phalanges $p_{i}$ and the rotation of the phalanges $\theta_{i}$ are shown.

proximal and distal phalanx, these equations were as follows:

$\left(\begin{array}{c}L_{0} \\ 0\end{array}\right)+\mathbf{R}_{\theta_{1}}\left(\begin{array}{c}p_{1} \\ t_{1}\end{array}\right)=\left(\begin{array}{c}X_{\mathrm{obj}} \\ Y_{\mathrm{obj}}\end{array}\right)+\mathbf{R}_{\theta_{1}}\left(\begin{array}{c}0 \\ -r_{\mathrm{obj}}\end{array}\right)$

$\left(\begin{array}{c}L_{0} \\ 0\end{array}\right)+\mathbf{R}_{\theta_{1}}\left(\begin{array}{c}L_{1} \\ 0\end{array}\right)+\mathbf{R}_{\theta_{1}} \mathbf{R}_{\theta_{2}}\left(\begin{array}{c}p_{2} \\ t_{2}\end{array}\right)=\left(\begin{array}{c}X_{\mathrm{obj}} \\ Y_{\mathrm{obj}}\end{array}\right)+\mathbf{R}_{\theta_{1}} \mathbf{R}_{\theta_{2}}\left(\begin{array}{c}0 \\ -r_{\mathrm{obj}}\end{array}\right)$

where index 1 and 2 denote the proximal and distal phalanx, respectively; $p_{i}$ is the contact point position on the phalanx; $t_{i}$ is the thickness of the phalanx; $\theta_{i}$ is the rotation of the phalanx; $\mathbf{R}_{\theta_{i}}$ is the rotation matrix; and $X_{\mathrm{obj}}$ and $Y_{\mathrm{obj}}$ is the position of the object with radius $r_{\mathrm{obj}}$.

The magnitude of the normal contact forces was calculated according to (Birglen et al., 2008, p. 65):

$F_{1}=-\frac{L_{1}\left(-p_{2}+h \cos \theta_{2}\right)}{p_{1} p_{2}\left(h+L_{1}\right)} \cdot T_{a}$

$F_{2}=\frac{h}{p_{2}\left(h+L_{1}\right)} \cdot T_{a}$

where

$h=c\left(\cos \theta_{2}-\psi-\sin \theta_{2}-\psi \cot \beta\right)$

with

$\cot \beta=\frac{c \sin \left(\theta_{2}-\psi\right) \sqrt{4 a^{2} b^{2}-N^{2}}+M\left(L_{1}+c \cos \left(\theta_{2}-\psi\right)\right)}{-\left(L_{1}+c \cos \left(\theta_{2}-\psi\right)\right) \sqrt{4 a^{2} b^{2}-N^{2}}+M c \sin \left(\theta_{2}-\psi\right)}$
Table 1. Design parameter used for DH-2.

\begin{tabular}{cccccccc}
\hline Parameter & $L_{0}$ & $L_{1}$ & $L_{2}$ & $a$ & $b$ & $c$ & $\psi$ \\
\hline Value $[\mathrm{mm}]$ & 30 & 60 & 40 & 20 & 60 & 10 & $105^{\circ}$ \\
\hline
\end{tabular}

with

$M=-L_{1}\left(L_{1}+2 c \cos \left(\theta_{2}-\psi\right)\right)+a^{2}-b^{2}-c^{2}$

$N=L_{1}\left(L_{1}+2 c \cos \left(\theta_{2}-\psi\right)\right)-a^{2}-b^{2}+c^{2}$

Simultaneously solving Eqs. (1) and (2) for each finger at a specific object position and object size resulted in the equilibrium configuration of the fingers $\left(p_{i}, \theta_{i}\right)$. Substituting this configuration in Eqs. (3) and (4) yielded the contact forces $F_{i}$. In the case of a negative proximal contact force, a new configuration was calculated where this phalanx had no contact with the object (i.e., Eq. (1) was omitted and $F_{1}=0$, Eq. (3)). A grasp equilibrium of the fingers with the object existed when the resultant of the contact forces is zero or when the fingers compress the object against the palm. The maximum allowable weight or force disturbance on the object was equal to the resultant of the contact forces pointing in the direction of the palm at the particular object position where this resultant force was maximal.

An iterative search was done to find the dimensions of the design parameters that would result in a hand that on the one hand could grasp the required range of object sizes and on the other hand was able to resist large disturbance forces. Based on this search the dimensions were chosen according to Table 1 , and the in-plane thickness $t_{i}$ of the phalanges were chosen as small as possible. The mechanical limits of the fingers were chosen $\theta_{1}=45^{\circ}-90^{\circ}$ and $\theta_{2}=0^{\circ}-90^{\circ}$ (Fig. 3), which allowed the hand to grasp all objects in the required range within the required closing and opening time.

In addition, if the actuation torque $T_{a}=1 \mathrm{Nm}$ would be applied to the fingers, the maximum allowable force disturbance on the object in the direction perpendicular to the palm (y-direction) would be at least $32 \mathrm{~N}$ and $5 \mathrm{~N}$ for the object with a diameter of 60 and $120 \mathrm{~mm}$, respectively. Since friction in the finger mechanism or between the object and fingers was not taken into account for these performance predictions, higher allowable forces can be expected for the real prototype.

\section{Materials and methods}

This section describes the experiments to assess the performance of the DH-2 with the maximum input voltage of $U_{\text {in }}=24 \mathrm{~V}$ applied to the motor. Furthermore, we will investigate the relation between $U_{\text {in }}$ and the contact forces. 


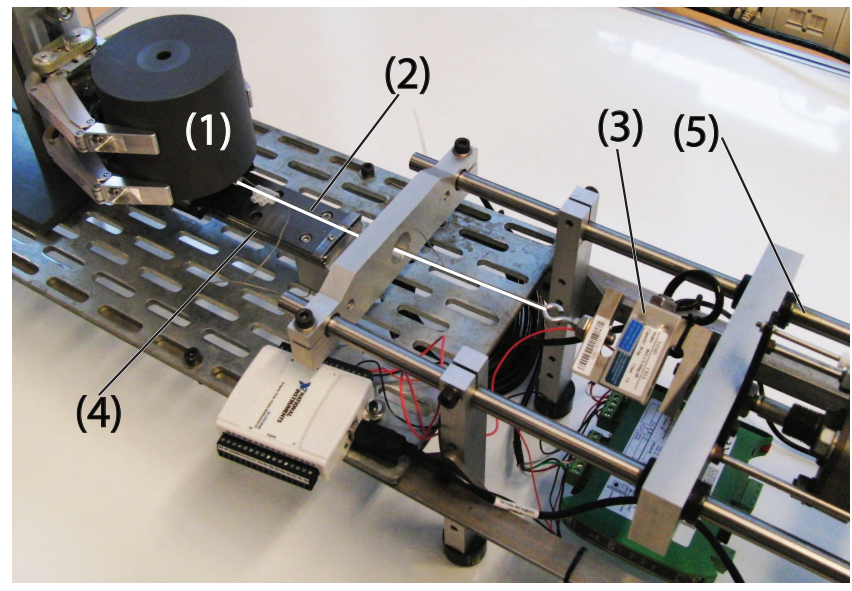

Figure 4. Setup for y-payload test where (1) is the test cylinder, (2) a cable (highlighted), (3) the load sensor, (4) the linear guidance for the object, and (5) the frame of the tensile tester.

\subsection{Payload}

The maximum payload of the hand depends on the size and shape of the object and the orientation of the hand with respect to gravity. In this study, the payload was determined for cylindrical objects with a diameter of 63,75 , and $110 \mathrm{~mm}$, while the hand was oriented with respectively the y-axis and Z-axis parallel to gravity (see Fig. 2).

The maximal payload parallel to the y-axis was measured by slowly pulling the grasped objects out of the hand in this direction and measuring the required force with the setup in Fig. 4. The objects were mounted on a horizontal linear guide (THK, RSR15WZM, friction force less than $0.5 \mathrm{~N}$ ), such that the objects could freely move along the y-axis. The objects were initially placed against the palm of the hand, while $U_{\text {in }}=24 \mathrm{~V}$ was supplied to the motor of the hand (i.e., the hand and object were in the grasp equilibrium configuration). Then the object was pulled out of the hand by a manually driven tensile tester at a speed of approximately $3 \mathrm{~mm} / \mathrm{s}$, while the required pull force was measured with a load cell (Zemic Europe, B3G-C3-50kg-6B, capacity $50 \mathrm{~kg}$ ), and the object position was measured using a LVDT (Schaevitz Sensors, 2000 LCIT). Each experiment was replicated 5 times. At least 1 min waiting time were kept between the experiments to have comparable motor temperatures at each experiment. To calculate the mean force as a function of the object displacement relative to the palm, the position where the pull force was $5 \mathrm{~N}$ was chosen as the zero position of the object. Then the measured pull force was re-sampled at a displacement interval of $1 \mathrm{~mm}$ by linear interpolation between the nearest measured positions.

For each object size, the maximum of the mean force was determined and regarded as the maximum payload $F_{\text {ymax }}$.

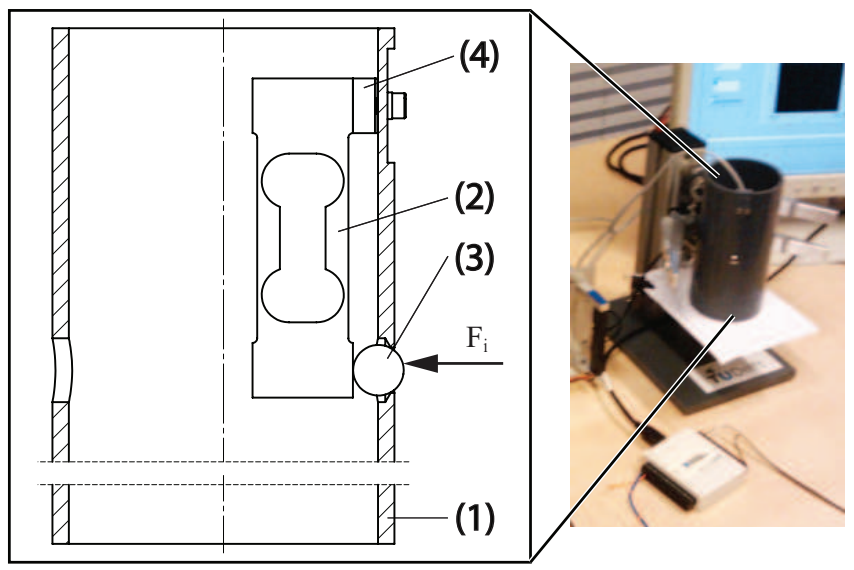

Figure 5. Overview of the test setup containing, (1) a PVC cylinder available in diameter $D_{\text {obj }}=63,75,110 \mathrm{~mm},(2)$ the load cell, (3) a steel ball, and (4) the sensor mount.

The maximal payload at an orientation parallel to the $\mathrm{z}$ axis depends on the friction between the fingers and the object:

$F_{\text {zmax }}=\sum F_{i} \cdot \mu_{i}$

where $\mu_{i}$ is the friction coefficient at the corresponding contact surface and $\sum F_{i}$ the sum of the contact forces when the object is in grasp equilibrium and the hand is actuated at $U_{\text {in }}=24 \mathrm{~V}$. The measurement of the contact forces applied to the three objects is described in the next section. Since the contact friction between the object and the fingers depends on their material properties, the minimum contact friction needed to hold objects of these sizes was calculated, assuming the required payload $F_{\text {zmax }}=20 \mathrm{~N}$.

\subsection{Input voltage - contact force relation}

The magnitude of the contact forces of the fingers on cylindrical objects of available diameter $D_{\mathrm{obj}}=63,75,110 \mathrm{~mm}$ was measured as a function of the motor input voltage $\left(U_{\text {in }}\right)$. The measurements were executed using the setup displayed schematically in Fig. 5. A load cell (HBM PW4MC3 $3 \mathrm{~kg}$ ) was mounted inside the cylinder. A steel ball was placed in a countersunk hole on the inside of the cylinder and was held in place by the sensor. A small part of the ball protruded through the hole of the cylinder to act as a contact point where the force could be measured. The cylinder was thus placed in the hand in such orientation that the contact force between the objects and the proximal phalanx $\left(F_{1}\right)$, distal phalanx $\left(F_{2}\right)$ and palm $\left(F_{\text {palm }}\right)$, respectively, could be measured on finger $\mathrm{A}$, assuming that this force is equal to the sum of the corresponding contact forces on the opposite fingers B and $\mathrm{C}$ (see Fig. 2). The cylinder was vertically supported by a platform on which the aforementioned orientations were plotted for better repeatability of the cylinder orientation. 
The load cell signal was amplified to a $\pm 10 \mathrm{~V}$ signal and A/D converted with a data acquisition unit (NI DAQ USB6008 ) resulting in a measurement resolution of 0.05 N. During the test three parameters were varied: (1) The input voltage $U_{\text {in }}$ of the motor, varying from $10 \mathrm{~V}$ limited by static friction of the mechanism to $24 \mathrm{~V}$ limited by the nominal voltage in steps of $2 \mathrm{~V}$, (2) The available object diameters $D_{\text {obj }}$ of $63 \mathrm{~mm}, 75 \mathrm{~mm}$ and $110 \mathrm{~mm}$, and (3) the contact point varying from the palm $F_{\text {palm }}$, the proximal phalanx $F_{1}$, and distal phalanx $F_{2}$. The full experiment was replicated 3 times. To check the linearity of the measured forces $F_{i}$ as a function of $U_{\text {in }}$, a linear regression analysis was applied to the experimental data using a least squares method. The data was fitted to the following line:

$F_{i}\left(U_{\text {in }}\right)=a \cdot\left(U_{\text {in }}-10 \mathrm{~V}\right)+b, \quad U_{\text {in }} \geq 10 \mathrm{~V}$

where $a$ is the slope and $b$ the $10 \mathrm{~V}$ intercept. The contact forces at the maximal input voltage $F_{i}\left(U_{\mathrm{in}}=24 \mathrm{~V}\right)$ was used to determine the maximal z-payload $F_{\text {zmax }}$ as mentioned in the previous section.

\subsection{Closing and opening time}

The time to fully close and open the hand was measured. A high-speed camera (Casio, EX-F1, 300fps) was used to record a full opening and closing cycle at an input voltage $U_{\text {in }}=24 \mathrm{~V}$. The opening and closing time was calculated as follows:

$\Delta t_{\mathrm{c}}=t_{\text {closed }}-t_{\text {open }}$

$\Delta t_{\mathrm{o}}=t_{\text {open }}-t_{\text {closed }}$

where $\Delta t_{\mathrm{c}}$ and $\Delta t_{\mathrm{o}}$ are the closing and opening time respectively, $t_{\text {closed }}$ the time when grasper was fully closed, and $t_{\text {open }}$ the time when the grasper was fully opened. This experiment was replicated 3 times and the mean closing and opening time was calculated.

\section{Experimental results}

Figure 6 shows the force-displacement characteristics to measure the maximal payload in y-direction for objects of diameter $D_{\text {obj }}=63,75$ and $110 \mathrm{~mm}$ respectively. Each individual measurement as well as the mean of the five repetitions are shown. The points $A=131.7 \mathrm{~N}, B=102.0 \mathrm{~N}$ and $C=26.3 \mathrm{~N}$ correspond to the mean maximal force $F_{\text {ymax }}$.

Figure 7 shows the contact forces measured at the proximal phalanx, distal phalanx and palm for each object size. The raw data $(*)$, the linear regression line, its formula $\left(F_{\text {reg }}\right)$ along with the individual $R^{2}$ values are shown in the respective subplot. The sum of the contact forces at $U_{\text {in }}=24 \mathrm{~V}$ for object sizes $D_{\mathrm{obj}}=63,75$ and $110 \mathrm{~mm}$ are 67.6, 63.6 and $63.6 \mathrm{~N}$ respectively, was obtained by the summation of two times the proximal phalanx force $\left(F_{1}\right)$, two time the distal phalanx force $\left(F_{2}\right)$, and the palm force $\left(F_{\text {palm }}\right)$. According to

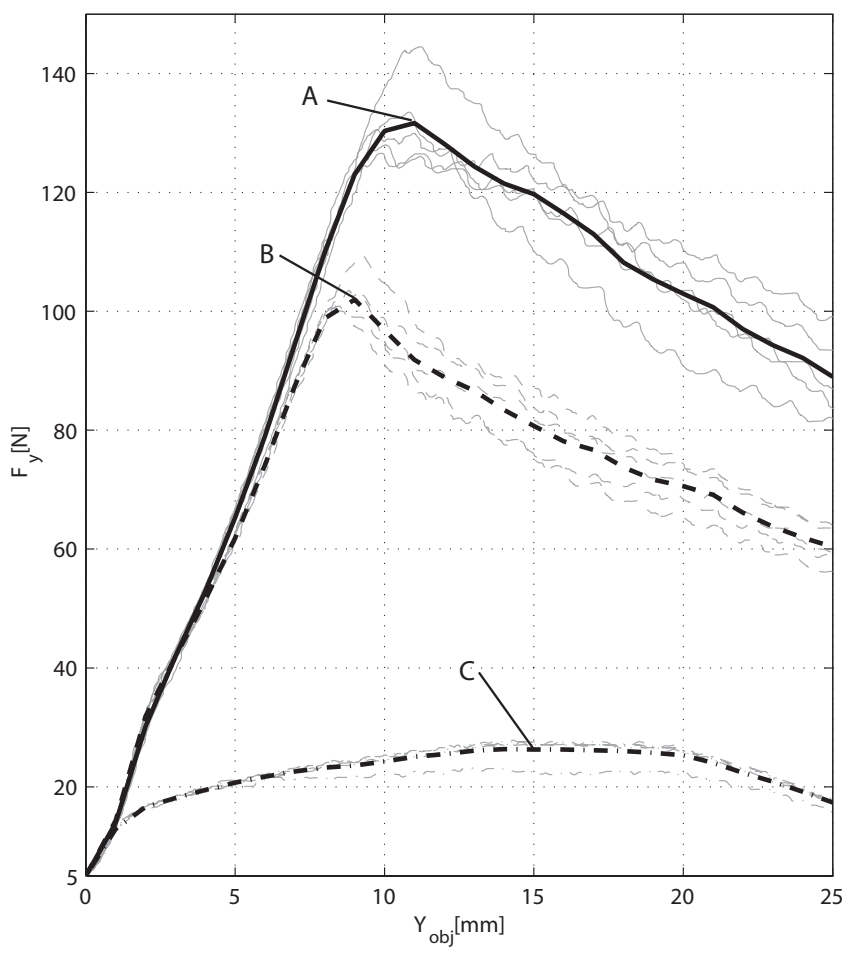

Figure 6. Results for the y-payload measurements where the solid line (-), dashed line (--) and dash-dotted line (-.-) are the mean forces $\left(F_{y}\right)$ on cylinders of $63,75,110 \mathrm{~mm}$ respectively, as a function of the $y$ displacement of the object $\left(Y_{\mathrm{obj}}\right)$. In gray the raw data is presented with using the same line type convention. $\mathrm{A}, \mathrm{B}$ and $\mathrm{C}$ denote the maximum of the mean force of the respective cylinder diameter.

Table 2. Closing and opening time of the hand in [s].

\begin{tabular}{lcccc}
\hline Repeat & 1 & 2 & 3 & Mean \\
\hline Closing time & 0.421 & 0.432 & 0.425 & 0.426 \\
Opening time & 0.450 & 0.432 & 0.430 & 0.437 \\
\hline
\end{tabular}

Eq. (9), the minimal required friction coefficients to achieve a payload of $20 \mathrm{~N}$ are thus $0.29,0.30$ and 0.36 , assuming that the contact friction is equal for all contact points. For cylinder diameter $110 \mathrm{~mm}$ the force on the proximal is zero because with these design parameters there is no 5 point grasp possible.

Table 2 shows the measured opening and closing times and their average. 

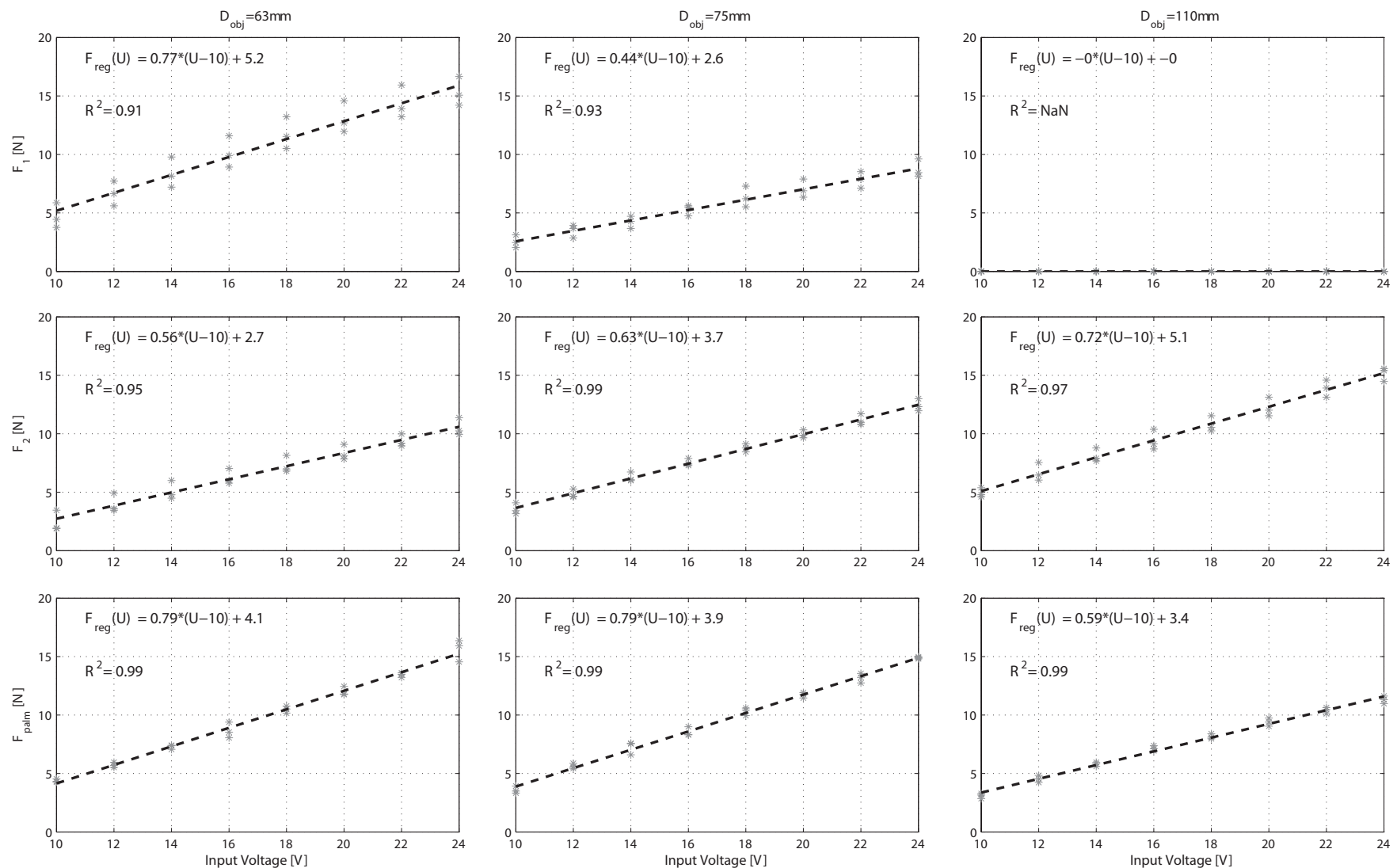

Figure 7. Results of the contact force measurements where, the stars $(*)$ are the raw data points, and the dashed line (--) the linear regressed test results, as function of the input voltage [V]. From left to right: cylinder diameter 63, 75 and 110 mm. From top to bottom: Contact forces on the proximal phalanx, distal phalanx, and palm.

Table 3. Comparison of the test results with the requirements where $F_{\text {ymax }}$ is the maximal force in y-direction, $\mu_{\min }$ the minimal required friction coefficient for $2 \mathrm{~kg}$ payload in $\mathrm{z}$-direction, $D_{\text {obj }}$ the object diameter, and $\Delta t_{i}$ the opening and closing time.

\begin{tabular}{lrlll}
\hline Criteria & Unit & Measured & \multicolumn{2}{c}{ Required } \\
\cline { 4 - 6 } & & & $\min$ & $\max$ \\
\hline$F_{\text {ymax }} \varnothing 63$ & {$[\mathrm{~N}]$} & 131.7 & 20 & - \\
$F_{\text {ymax }} \varnothing 75$ & {$[\mathrm{~N}]$} & 102.0 & 20 & - \\
$F_{\text {ymax }} \varnothing 110$ & {$[\mathrm{~N}]$} & 26.3 & 20 & - \\
\hline$\mu_{\min } \varnothing 63$ & {$[-]$} & 0.29 & - & - \\
$\mu_{\min } \varnothing 75$ & {$[-]$} & 0.30 & - & - \\
$\mu_{\min } \varnothing 110$ & {$[-]$} & 0.36 & - & - \\
\hline$D_{\mathrm{obj}}$ & {$[\mathrm{mm}]$} & 63 to 110 & 60 & 120 \\
\hline$\Delta t_{\mathrm{c}}$ & {$[\mathrm{s}]$} & 0.426 & - & 0.500 \\
$\Delta t_{\mathrm{o}}$ & {$[\mathrm{s}]$} & 0.437 & - & 0.500
\end{tabular}

\section{Discussion}

The requirements to the DH-2 were a simple and robust design that was able to grasp objects of a payload of $2 \mathrm{~kg}$ and of a size between $\varnothing 60$ to $\varnothing 120 \mathrm{~mm}$ within $0.5 \mathrm{~s}$ closing or opening time. In this paper, the performance was assessed. In Table 3 all measured values are compared with there corresponding requirements. Though the maximal payload perpendicular to the palm (parallel to the y-axis) is strongly decreasing for increasing object size, the minimal disturbance force requirement is satisfied for the measured object sizes. The payload parallel to the palm (in z-direction) is less dependent on the object size but greatly depends on the friction between the object and the fingers. This friction coefficient has to be greater then 0.36 for the largest object. This means that when the payload reaches critical values, friction coating or synthetic phalanges is necessary, but for lower payloads blank metal phalanges suffices. The friction enhancements can also be unevenly distributed over the contact points if this would be more convenient. The tested object sizes do not cover the full required range of objects sizes. However, the benchmark test in Kragten et al. (2010) showed that the DH-2 can grasp objects between $\varnothing 55$ to at least $\varnothing 120 \mathrm{~mm}$ in 
a power grasp configuration. The closing and opening time satisfy the required $0.5 \mathrm{~s}$.

It was chosen to design the hand without any force or position sensors, to keep the hand cheap and less vulnerable. The motor input voltage was chosen to control the magnitude of the contact forces. The relation between the input voltage and the contact forces was measured. Applying the linear regression to the contact force measurement resulted in a regression coefficient $R^{2}>0.9$ indicating a strong linear relationship. In addition, we observed that the sum of the contact forces on the object hardly changed for different object sizes. This means that the input voltage of the motor seems suited for open-loop control of the grasp forces of the hand.

Comparing the design of the DH-2 with the DH-1, we observed that the choice of gears and belt transmissions instead of a cable-pulley transmission greatly improved the reliability of the design. The main dimensions of the fingers of the DH-2 also changed based on performance calculations. The DH-2 can grasp larger object sizes compared to the DH-1, while the total length of the fingers remained constant.

\section{Conclusions}

This paper revealed the design choices to develop a cheap, simple and robust underactuated robotic hand for industrial purposes and showed the resulting prototype. Tests to assess the payload, closing time and graspable range showed that: (1) A minimal payload of $2 \mathrm{~kg}$ can be grasped, providing that the contact friction is at least $0.36,(2)$ the hand can be closed or opened within $0.5 \mathrm{~s}$, and (3) objects sizes from $\varnothing 55$ to $\varnothing 120 \mathrm{~mm}$ can be held in a power grasp configuration. Hence, the DH-2 satisfies the main requirements provided by industrial partners.

It was shown that the magnitude of the contact forces can be controlled by open-loop control on the input voltage on the motor, making it possible to leave out force sensors and electronics needed for closed-loop control.

The key factors that lead to a commercially attractive, functional and reliable underactuated hand were (1) the choice of a simple mechanism consisting of conventional linkages, gears and belt transmissions; (2) the use of a minimal amount of motors and sensors; and (3) a dimensional design based on performance predictions by a grasp model.
Acknowledgements. We gratefully acknowledge the help of Tim Krijger with the contact force measurements and Gerwin Smit for the use of the tensile tester for the payload measurement.

Edited by: J. L. Herder

Reviewed by: two anonymous referees

\section{References}

Birglen, L., Laliberté, T., and Gosselin, C.: Underactuated robotic hands, Vol. 40, Springer Tracts in Advanced Robotics, Springer Verlag, Berlin, Heidelberg, 2008.

Kragten, G. and Herder, J.: Ability to hold grasped objects by underactuated hands: performance prediction and experiments, Mech. Mach. Theory, 45, 408-425, 2010.

Kragten, G., Meijneke, C., and Herder, J.: A Proposal of Benchmark Tests for Underactuated or Compliant Hands, Mech. Sci., in press, 2010.

Laliberté, T. and Gosselin, C.: Underactuation in space robotic hands, in: Proceedings of the 6th International Symposium on Artificial Intelligence and Robotics \& Automation in Space, 2001.

Meijneke, C. and Wilbers, F.: Slimme Robothanden, Mikroniek, 5-8, 2009 (in Dutch).

Napier, J.: The prehensile movements of the human hand, J. Bone Joint Surg., British Volume, 38, 902-913, 1956.

Townsend, W.: The BarrettHand grasper- programmably flexible part handling and assembly, Ind. Robot, 27, 181-188, 2000. 